\title{
A Carreira Médica e os Fatores Determinantes da Saída do Serviço Nacional de Saúde
}

\section{The Medical Career and the Key Factors Driving the Exodus of Doctors from the National Health Service in Portugal}

\author{
Marianela FERREIRA $\square^{1,2}$, Alexandra LOPES ${ }^{3,4}$, Miguel GUIMARÃES ${ }^{5,6}$, Henrique BARROS ${ }^{1,7}$ \\ Acta Med Port 2018 Sep;31(9):483-488 - https://doi.org/10.20344/amp.10121
}

Estudo desenvolvido no Instituto de Saúde Pública da Universidade do Porto e no Instituto de Sociologia da Universidade do Porto, em colaboração com o Gabinete de Estudos da Secção Regional do Norte da Ordem dos Médicos (SRNOM).

\section{RESUMO}

Introdução: Este artigo aborda a temática da organização e gestão da carreira médica em Portugal no quadro do Serviço Nacional de Saúde. Foca, em particular, alguns indicadores de motivação e satisfação profissional.

Material e Métodos: Este artigo resulta de um projeto de investigação mais alargado sobre a organização da carreira médica em Portugal. Foram aplicados inquéritos a médicos especialistas ativos, a realizar o internato de especialidade médica e que já abandonaram o Serviço Nacional de Saúde. A base de sondagem compreende os médicos inscritos na Secção Norte da Ordem dos Médicos.

Resultados: Foram inquiridos 3253 médicos. Os níveis de satisfação dos médicos variam em função da idade, do tipo de ligação ao SNS e associados às expectativas em relação ao futuro profissional.

Discussão: Apesar da estreita ligação com o Serviço Nacional de Saúde, os médicos mais jovens evidenciaram maiores níveis de insatisfação profissional e incerteza em relação ao seu futuro profissional. Ainda que com graus de descontentamento variáveis em função dos grupos considerados, a insatisfação dos profissionais inquiridos é com as condições materiais de exercício da profissão e com as oportunidades de progressão. No plano das relações interpessoais e da formação, a satisfação é elevada. A antecipação da reforma e a transferência para o setor privado são opções que os profissionais equacionam como estratégia para responder à insatisfação profissional.

Conclusão: O estudo demonstra que as configurações atuais de funcionamento do Serviço Nacional de Saúde não diminuem o interesse pelo exercício da medicina, mas sim motivam a procura de condições mais aliciantes e compensatórias no exercício da mesma.

Palavras-chave: Aposentadoria; Medicina Estatal; Médicos; Motivação; Satisfação no Emprego; Setor Privado

\section{ABSTRACT}

Introduction: This article addresses the organization and management of medical careers in Portugal within the framework of the National Health Service. It focuses, in particular, on some indicators of motivation and professional satisfaction.

Material and Methods: This article is part of a broader research project on the organization of medical careers in Portugal. It draws on the results of a survey carried out to active medical doctors, residents and those that have already abandoned the National Health Service. The sample was drawn from the database of physicians enrolled in the Northern Section of the Medical Association.

Results: A total of 3253 physicians were surveyed. Physician satisfaction levels vary according to age, type of connection to the National Health Service and are associated with expectations regarding the professional future.

Discussion: Despite the close connection with the National Health Service, younger doctors showed higher levels of professional dissatisfaction and uncertainty regarding their professional future. Although with varying degrees of discontent depending on the groups considered, the dissatisfaction of the professionals interviewed is with the physical conditions of the workplace and with the opportunities for career advancement. At the level of interpersonal relations and training, satisfaction is high. The anticipation of retirement and the transfer to the private sector are options that professionals consider as a strategy to respond to professional dissatisfaction.

Conclusion: The study shows that the current functioning of the National Health Service does not diminish the interest in the practice of medicine, but rather motivates the search for more attractive and rewarding working conditions.

Keywords: Job Satisfaction; Motivation; Physicians; Private Sector; Retirement; State Medicine

\section{INTRODUÇÃO}

A necessidade de sustentabilidade financeira do Serviço Nacional de Saúde (SNS), num quadro de contenção financeira e de diminuição do financiamento público, deba- te-se com as crescentes exigências de elevados níveis de qualidade e cobertura na prestação de cuidados de saúde (Simões; Campos, 2014) O agravamento da fragilidade

\footnotetext{
1. Faculdade de Medicina. Universidade do Porto. Porto. Portugal.

2. Unidade de Investigação em Epidemiologia. Instituto de Saúde Pública da Universidade do Porto. Porto. Portugal.

3. Faculdade de Letras. Universidade do Porto. Porto Portugal.

4. Coordenadora do Instituto de Sociologia da Universidade do Porto. Porto. Portugal

5. Bastonário. Ordem dos Médicos. Lisboa. Portugal.

6. Serviço de Urologia. Centro Hospitalar de São João. Porto. Portugal.

7. Presidente. Instituto de Saúde Pública da Universidade do Porto. Porto. Portugal.

$\bowtie$ Autor correspondente: Marianela Ferreira. marianela.f333@gmail.com

Recebido: 20 de dezembro de 2017 - Aceite: 04 de julho de 2018 | Copyright @ Ordem dos Médicos 2018
} 
Ferreira M, et al. A carreira médi
Caracterização socioprofissional

Seguindo a tendência de feminização da profissão médica, mais de metade dos médicos em exercício no SNS que foram inquiridos na primeira fase do estudo eram mulheres $(59,5 \%)$. A grande maioria dos médicos conclui uma licenciatura ou um mestrado integrado $(87,2 \%)$, portanto os graus exigidos para o exercício da medicina de acordo com o modelo educativo en vigó à data da conclusão do grau académico. A parc

O facto de terem sido inquiridos médicos no início e no fim da carreira justifica a grande amplitude etária verificada: fim da carreira justifica a grande amplitude etária verificada: $31,0 \%$ tinham entre os 31 e os 40 anos e $32,4 \%$ entre os
51 e 60 anos. Em consonância com esta distribuição etária, 51 e 60 anos. Em consonância com esta distribuição etária,

cerca de um terço começou a exercer medir 1980 e outro terço entre 2001 e 2010.

Tendo por base o definido na Carreira Especial Médica, conforme estabelecido no Decreto-Lei n. ${ }^{\circ}$ 177/2009, de 14 de agosto, $57,4 \%$ dos médicos tinha como qualificação médica o grau de especialista e $40,6 \%$ o de consultor; sobre a categoria na carreira, a maioria dividia-se entre a de assistente $(44,3 \%)$ e de assistente graduado (42,1\%). Sobre o tipo de vínculo contratual com o SNS, $61,3 \%$ tinham contrato de trabalho em funções públicas e $38,7 \%$ um contrato individual de trabalho. Perto de um quinto exercia funções de direção/chefia $(22,8 \%)$, sobretudo de direção de serviço $(41,1 \%)$ ou de outras unidades funcionais $(29,1 \%)$.

Dois terços dos médicos exerciam medicina na área hospitalar $(65,2 \%)$ e um terço na de medicina geral e familiar $(32,3 \%)$, tendo as restantes áreas (saúde pública e medicina legal e medicina do trabalho) um peso residual. Esta distribuição por áreas de exercício profissional é reflexo da manutenção da centralidade dos hospitais na organização do SNS, não obstante a recente aposta na rede de cuidados primários.

Ao longo da sua carreira profissional a generalidade dos médicos manteve uma relação próxima ao SNS, na medida em que $86.9 \%$ destes indicou sempre ter trabalhamedida en que mais de dois terços não o fazia em regime de dedicação exclusiva $(69,1 \%)$ e mais de metade acumulava funções no setor privado de saúde $(54,4 \%)$. Alguns médicos indicaram desenvolver outras atividades além do exercício da medicina, essencialmente de docência $(18,8 \%)$, investigação

cina, essencialmente de docência) e consultadoria $(5,5 \%)$.

Os médicos que trabalhavam nos serviços de urgência, externa e interna, unidades de cuidados intensivos e unidades de cuidados intermédios faziam-no, sobretudo, em períodos de 12 ou 24 horas (14,2\% e 15,3\%, respetivamente). Perto de metade dos médicos não trabalhavam naqueles serviços $(46,9 \%)$.

\section{Dimensões de satisfação profissional}

É marcada a insatisfação dos médicos com as jornadas a menor disponibilidade de tempo para outras atividades extralaborais. Somadas as percentagens dos médicos que se manifestaram insatisfeitos ou muito insatisfeitos, $60,5 \%$ dos médicos mostraram o seu descontentamento com numero de horas trabalhadas e $74,1 \%$ com o tempo disponível para a família, amigos e atividades de lazer/sociais. Apesar da normal contratualização de determinado número de horas de trabalho no SNS (40 horas por semana para $52,8 \%$ dos médicos, dividindo-se os restantes entre as 35 e as 42 horas), $25,0 \%$ referiram que ultrapassavam o seu horário de trabalho mais do que uma vez por semana ou praticamente todos os dias. Cerca de metade dos médcos ter indicado que os descansos compensatóris medpor lei teria direito não são cumpridos ( $46,6 \%$ ) ou que des-

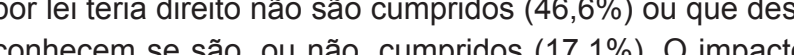
durã̃o das jomad 作 poder ser dissociado do facto de $39,3 \%$ dos medicos ter dicado a pressáo do trabalholexaustao como determinan-

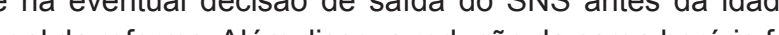

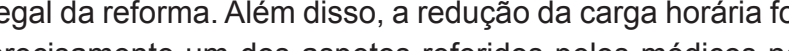
precisamente um dos aspetos referidos pelos médicos $n$ ativo $(31,2 \%)$ que os poderiam levar a protelar a transição reforma e, assim, a saída do SNS.

Também a remuneração se revelou um aspeto relevante na avaliação da satisfação profissional dos médicos: $76,7 \%$ mostraram-se insatisfeitos, ou muito insatisfeitos, com rendimento que auferem do seu trabalho no SNS que, para cerca de metade dos médicos, é inferior a $3000,00 €$ brutos mensais $(46,9 \%)$

A apreciação das oportunidades de carreira na especialidade dividiu opiniões: cerca de $28 \%$ indicou concordar e a mesma parte discordar com o facto de terem tido boas oportunidades de carreira; $23,2 \%$ não concordaram nem discordaram. Já em relação às futuras oportunidade profissionais, $62,5 \%$ indicou discordar (nalguns casos totalmente), da existência de boas perspetivas de carreira $\mathrm{n}$ sua especialidade médica.

Sobre a participação na tomada de decisões, os resulados obtidos indicam alguma divisão entre a satisfação $e$ insatisfacão, com 50,1\% dos médicos a mostrarem-se descontentes (somando os insatisfeitos com os muito insatisfeitos), com 19,4\% para quem este era um aspeto indiferente e com $30,5 \%$ satisfeitos ou muito satisfeitos.

É na dimensão relacionamento com os colegas de profissão que os médicos no ativo no SNS se mostraram lobalmente satisfeitos $(76,6 \%$ mostraram-se satisfeitos ou muito satisfeitos).

\section{Cenários de saída do SNS}

Os médicos no ativo no SNS mostraram-se divididos na intenção de saírem do SNS por reforma, antecipada ou ão.

A maior parte dos médicos considera sair por reforma na idade legalmente definida para o efeito, isto é, quando atingir os 66 anos $(41,6 \%)$ ou os 65 anos $(18,4 \%)$. É de notar, contudo, a vontade de $15,3 \%$ dos médicos de proongarem a sua atividade até aos 70 anos, o limite máximo possível na função pública. Para $45 \%$ dos inquiridos, porém, a possibilidade de antecipar a reforma é algo que 
Ferreira M, et al. A carreira médica e os fatores detete

Quanto à possibilidade de emigração para exercício da medicina fora de Portugal $66,1 \%$ afastam liminarmente essa opçâo, dividindo-se os restantes entre os $17,3 \%$ uma possibilidade e os $17,6 \%$ que o veem como uma possibilidade.

No que diz respeito à transferência para o setor privad de saúde em Portugal, são $48,3 \%$ aqueles que afastam de forma clara esse cenário como cenário de desenvolvimento profissional futuro. Os restantes inquiridos dividem-se entre os 33,5\% que não têm a certeza de essa ser uma hipótese ue considerariam e os $18,2 \%$ que a vislumbram como algo que acontecerá com grande certeza.

\section{Médicos que saíram do SNS}

\section{Caracterização socioprofissiona}

No caso dos médicos inquiridos que já tinham saído do SNS, uma ligeira maioria eram homens $(55,1 \%)$. As média etárias foram significativamente distintas em função do motivo de saída: cerca de 68 anos para os que optaram pela reforma, 50 anos para os que optaram pelo setor privado de saúde e 40 anos para os que decidiram emigrar.

No caso dos médicos a realizar internato a média de dade foi de aproximadamente 31 anos.

A saída dos médicos do SNS aconteceu sobretudo porque decidiram reformar-se $(43,3 \%)$ ou porque optaram pelo trabalho no setor privado de saúde $(36,1 \%)$. Os casos de opção pela emigração para exercer medicina no estrange ro foram residuais $(7 \%)$

Independentemente do motivo de saída do SNS, a grande maioria dos médicos sempre trabalhou no SNS, ainda que cerca de metade, em todos os casos, não estitaram por sair do SNS para o setor privado, $83,6 \%$ já a ontes taram por sair do SNS para o setor privado, 83,6\% ja antes foi de $73,3 \%$ para os que sairam por reforma e apenas de $36,7 \%$ para os que saíram por emigração.

Um outro aspeto distintivo com base no motivo de saída do SNS é o tipo de vinculo contratul: enquanto $92,7 \%$ dos que se reformaram tinham um contrato de trabalho em funções públicas, nos restantes casos a percentagem desce para cerca de $50 \%$. Também os que se reformaram tinham sobretudo um regime laboral semanal de 42 horas $(47,9 \%)$ enquanto que entre os restantes é mais comum o regim de 35 horas para os que optaram pelo privado $(41,5 \%)$ e de 40 horas para os que emigraram $(59,2 \%)$.

Foi entre os médicos que saíram por reforma que se registaram mais casos de exercício de funções de chefia $(52,5 \%)$. O exercício destas funções entre os restantes me dicos foi muito menos expressivo: $79,8 \%$ dos que saíram para o setor privado e $98,9 \%$ dos médicos que emigraram não exerciam funções de chefia no SNS.

Dimensões da satisfação profissional

A maioria dos médicos que saíram do SNS declararam grande satisfação com o relacionamento com os colegas quando ainda trabalhavam no SNS. Em qualquer dos cenáou muito satisfeitos com aquela dimensão rondou os $70 \%$. Também a frequência de ações de atualização científica foi um aspeto avaliado com níveis de satisfação variável entre os três grupos: entre os médicos reformados, foram $50 \%$ os que se mostraram satisfeitos; entre os que optaram pelo setor privado apenas $40 \%$ e, finalmente, entre os médicos que emigraram apenas $30 \%$.

No que diz respeito à dimensão progressão na carreira, de novo é identificada variação associada aos três grupos. No grupo dos que saíram por reforma, os satisfeitos ou muito satisfeitos são $56,7 \%$, enquanto que entre aqueles que saíram para o setor privado essa proporção é de $16,6 \%$ e entre os que emigraram é de $6,1 \%$.

Em relação aos restantes aspetos é evidente uma diferenciação entre os médicos que saíram por reforma - que se mostraram divididos entre a satisfação e a insatisfação, e os médicos que optaram pelo setor privado ou pela emigração e que, no conjunto, se mostraram sobretudo insatistellos com aquelas dimensōes, particularmente estes últimos. No caso dos médicos que optaram pelo setor privado e dos que emigraram, a insatisfação foi particularmente evidente com a remuneração e o tempo disponível para a família. Nos dois casos, o somatório dos insatisfeitos com os muito insatisfeitos foi superior a $50 \%$. Ainda assim, as restantes dimensões foram avaliadas negativamente, alcançando sempre percentagens de insatisfação em torno dos $50 \%$ se somadas as duas categorias. Apenas no caso da dimensão condiçõos físicas e equipamentos se contaram cerca de $1 / 3$ de médicos satisfeitos.

Quando é pedida uma apreciação global do SNS, o sentido das diferenças entre os grupos mantém-se alinhada como as tendências sinalizadas em cada dimensão. Entre os que saíram por reforma são $15,5 \%$ os insatisfeitos ou muito insatisfeitos, $32 \%$ os indiferentes e $47,2 \%$ os satisfeitos ou muito satisfeitos. Estas proporç̃̃es para o grupo dos que sairam para o setor privado são, respetivamente, emigração, as proporções são iguais a $67,4 \%, 16,3 \%$ e $8,1 \%$

Médicos a realizar o internato de especialidade médica Foi pedido a cada inquirido, médicos a realizarem $\mathrm{o}$ internato de especialidade, que indicassem o seu grau de satisfação com um conjunto de dimensões relativas ao acesso ao internato. Os resultados médios, numa escala de 1 a 5, em que 5 corresponde ao nível máximo de satisfação, foram, por ordem decrescente (desvio-padrão entre parêntesis): metodo de divulgação da lista de vagas de especialidade com média de 3,24 (1,21); método de divulgação da lista de colocados com média de 3,16 (1,12); locais para a escolha com média 3,01 (1,17); momento do ano para a escolha com média igual a 2,98 (1,20); periodo disponível para a escolha da área com media 2,67 $(1,20)$, e distribuição de vagas por especialidade com média 2,52 (1,15).

Quando inquiridos sobre a perspetiva futura de ficarem no SNS após conclusão da especialidade médica, $42,9 \%$ declararam não saber o que vai acontecer, sendo $35,3 \%$ os que acreditam que isso ira acontecer e $18,1 \%$ os que não acreditam ficar no SNS.

Ficar na área de residência após conclusão do internato não se revelou um aspeto relevante para mais de metade dos médicos que 0 avaliaram como nada importante $(59,0 \%)$.

Cerca de metade dos médicos internos equacionava a possibilidade de exercer medicina no estrangeiro depois de terminar o internato de especialidade $(47,2 \%)$. Dos restantes, $37,1 \%$ indicou não saber se permanecerá no país e apenas $11.4 \%$ indicou que nenhum valor remuneratório o faria sair de Portugal.

\section{DISCUSSÃO}

Todos os médicos inquiridos mostraram uma estreita ligação com o SNS, não só enquanto local de formação durante o internato de especialidade como, depois, ao longo da sua trajetória profissional. Contudo, enquanto esta ligação parece mais evidente e sólida entre os médicos mais velhos, os mais jovens revelam já elevados níveis de incerteza sobre a continuidade ou não no SNS, sobretudo os que se encontram atualmente a realizar o internato de especialidade. A insatisfação com as condições atuais de exercicio da medicina e a incerteza sobre as oportunidades futuras leva a que estes médicos procurem outras alternativas ao SNS. Exemplo disso é a acumulação de funções no setor privado de saúde, sobretudo entre os que optaram pela saída para exercício naquele setor. No caso dos médicos que optam por sair do SNS, as opções passam essencialmente pela reforma, antecipada, ou não, e, nos casos em que os médicos não reúnem condições para isso ou simplesmente não o pretendem fazer, pela decisão de transitar em exclusivo para o setor privado de savide ou, então, sobretudo entre os mais novos, pela procura de novas oportunidades de exercio da medicina fora de Portugas. oportich cício de medina, cicio da mécis

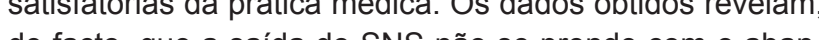
de facto, que a saica mo SNs náo se prende com o abandono do exercicio da medicina, mas sim com a procura de condiçoes mais aliciantes e compensatórias no exercicio da atividade médica. Em qualquer das situaçôes aumenta o risco de saida de médicos no SNS e, nos casos de emigração, dos recursos disponiveis no pais. A diminuição dos médicos em exercicio no SNS e do potencial daqueles que o poderão integrar (no caso dos internos) terá necessariamente implicaçôes na eficiência e sustentabilidade do SNS, bem como na qualidade dos serviços de saúde prestados. ${ }^{5}$ Esta situação podera ainda implicar um maior esforço e sobrecarga dos médicos que permanecem no SNS e, por isso, potenciar situações de burnout, abandono e desmotivação. ${ }^{6}$ Estudos sobre a satisfação profissional dos médicos revelam existir uma relação entre a sua satisfação profissional e a qualidade dos serviços de saúde prestados que, contudo, carece de mais investigações e profundamento. ${ }^{7}$

Independentemente do motivo de saída do SNS, a maioria dos médicos revelou elevados niveis de satisfaçẫo com o relacionamento com os colegas e com a frequência e ações de atualização científica. Comum à maioria dos médicos foi também a apreciação negativa de dimensões específicas do funcionamento do SNS, em concreto com tempo de trabalho realizado, as oportunidades de progressão na carreira e a remuneração, ainda que com graus de descontentamento variáveis em função dos grupos considerados. Foram sobretudo os médicos mais novos e os que optaram por sair do SNS (por emigracão) os que mostram maior insatifaça situac̃ão que se poderá justificar pelas difentes condicões de excício da medicina des expestivas de desencesimento profission destes médicos face aos mais velhos e que tiveram opoturides de

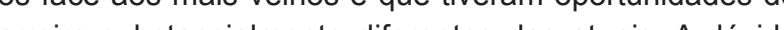
cos

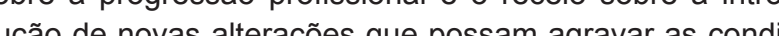
ç

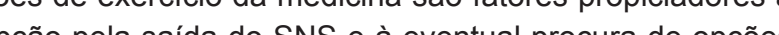
mais motivadoras de exercício da medicina. Apesar disso, insatisfacão dos diferentes grupos de médicos com os

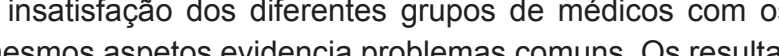
列 dos oblidos evidenciam a importância de atuar ao nivel da NS (ção das jornadas de trabalho reais dos médicos no (que, segundo a informação recolhida, são substancalmente superiores às contratualizadas), situação potencalmente agravada pelo não cumprimento dos periodos de descanso, o que poderá ter um duplo efeito: na diminuição cos em exercício no SNS e na diminuição do risco de saída do SNS, independentemente do motivo.

Será também importante considerar a insatisfação com s oportunidades de carreira e desenvolvimento profissional, sobretudo notada entre os médicos mais jovens que revelaram baixas expetativas sobre as oportunidades de desenvolvimento profissional no SNS a curto e a médio prazo, situaça que se podéá constituir como fator prepondera privado ou por emigração, na procura de condicões setor de condições mais

No caso da remuneração, esta mostrou-se particularmente significante nas decisões de saída tomadas pelo medicos que optaram por trabalhar exclusivamente no setor privado de saúde ou pela emigraçăo. De facto, os dados obtidos demonstram que estes dois cenarios foram estaisticamente significantes na sua associação à insatisfação profissional, sobretudo se considerada a dimensão remuneração. No caso dos medicos que optaram pela emigração, também as dimensões participação na tomada de decisão, carga de trabalho em horas e perspetivas de carreira se mostraram significantes. Situação diferente é a verificada no caso dos médicos que optaram pela reforma cuja opção de saida não se mostrou estatisticamente significante $\mathrm{na}$ associação à insatisfação profissional. Um estudo anterior mostrou que a saída dos médicos mais velhos acontece 
sobretudo por discordância com o enquadramento legal de aposentação e exposição a situações de discriminação etária negativa. ${ }^{1}$

\section{CONCLUSÃO}

Todos os médicos inquiridos mostraram uma estreita ligação com o SNS, não só enquanto local de formação durante o internato de especialidade como, depois, ao longo da sua trajetória profissional. São sinalizadas variações geracionais que remetem para as próprias dinâmicas de mudança e ajustamento do SNS ao longo das últimas décadas.

Ainda que com graus de descontentamento variáveis em função dos grupos considerados, a insatisfação dos profissionais inquiridos é com as condições materiais de exercício da profissão e com as oportunidades de progressão. No plano das relações interpessoais e da formação, a satisfação é elevada. A antecipação da reforma e a transferência para o setor privado são opções que os profissionais equacionam como estratégia para responder à insatisfação profissional. O estudo demonstra que as configurações atuais de funcionamento do SNS não diminuem o interesse

\section{REFERÊNCIAS}

1. Ferreira M. Sair Bem. Médicos e Enfermeiros nos Trajectos Finais das suas Vidas Profissionais. Lisboa: Coisas de Ler; 2015.

2. Fonseca A. Desenvolvimento psicológico e processos de transiçãoᄀ adaptação no decurso do envelhecimento. In: Paúl C, Ribeiro O, editores. Manual de Gerontologia. Lisboa: Lidel; 2012. p 95-106.

3. Munnel AH, Sass SA. Working Longer. The Solution to the Retirement Income Challenge. Washington: Brookings Institution Press; 2008.

4. Davidson JM. Retirement intentions of doctors who qualified in the United Kingdom in 1974: postal questionnaire survey. J Pub Health Med. 2001; 23: 323-8. pelo exercício da medicina, mas sim motivam a procura de condições mais aliciantes e compensatórias no exercício da mesma.

\section{PROTECÇÃO DE PESSOAS E ANIMAIS}

Os autores declaram que os procedimentos seguidos estavam de acordo com os regulamentos estabelecidos pelos responsáveis da Comissão de Investigação Clínica e Ética e de acordo com a Declaração de Helsínquia da Associação Médica Mundial.

\section{CONFIDENCIALIDADE DOS DADOS}

Os autores declaram ter seguido os protocolos do seu centro de trabalho acerca da publicação de dados.

\section{CONFLITOS DE INTERESSE}

Os autores declaram não terem qualquer conflito de interesse relativamente ao presente artigo.

\section{FONTES DE FINANCIAMENTO}

Os autores declaram não ter recebido subsídios ou bolsas para a elaboração do artigo.

5. Landon BE, Reschovsky JD, Pham HH, Blumenthal D. Leaving Medicine: the consequences of physician dissatisfaction. Medical Care. 2006; 44: 234-42.

6. Aasland OG, Rosta J, Nylenna M. Healthcare reforms and job satisfaction among doctors in Norway. Scand J Public Health. 2002; 38: 25-38.

7. Franco LM, Bennett S, Kanfer R. Health sector reform and public sector health worker motivation: a conceptual framework. Soc Sci Med. 2002; 54: $1255-66$. 